\title{
SPECTRA OF QUASARS WITH EXTREME CONTINUUM
}

\section{PROPERTIES}

\author{
SMITA MATHUR \\ Harvard-Smithsonian Center for Astrophysics, Cambridge, MA 02138, USA
}

\begin{abstract}
The quasar population as a whole covers a wide range of continuum properties; sufficient to cause a marked difference in the physical conditions of the gas in the nuclear region of a quasar. In this paper we will discuss our results on 3C351, an X-ray "quiet" quasar; and 3C212, a "red" quasar. We identify the "warm absorber" observed in the ROSAT observations of 3C351 to be the UV absorber observed by HST.
\end{abstract}

\section{Introduction}

The quasar population covers a wide range of continuum shapes ('SEDs', see Elvis, these proceedings). One consequence is that photoionization models must reflect the difference in the physical conditions of the line emitting gas; so using an "average quasar" obscures the physics. Multiwavelength (IR-X-ray) observations can now be gathered for a wide range of quasars; so photoionization theory can be applied to individual objects self-consistently. Here we will discuss two extreme cases-one with 'no' X-ray and one with 'no' UV, ionizing photons to look for the governing parameters in BELR physics

\section{3C351: An X-ray Quiet Quasar}

3 C351 is $\sim 10$ times less X-ray bright than a typical radio loud ( $\left.\alpha_{o x}=1.4\right)$ quasar, so we call it 'X-ray quiet' (Figure 1a). Quasi-simultaneous optical (MMT) and Xray (ROSAT) observations rule out variability as a cause of the low X-ray flux. The ROSAT X-ray spectrum of the object (Fiore et al, 1993) was not well fit with a simple power-law, with the residuals strongly suggesting an Oxygen edge at $0.76 \pm$ $0.08 \mathrm{keV}$. A 'warm' (ionized) absorber along the line of sight instead gives a good fit with a column density, $N_{H}=1.4 \pm 0.3 \times 10^{22} \mathrm{~cm}^{-2}$; and an ionization parameter, $U=6.7_{-1.2}^{+2.3}$. The large $U$ of the absorber implies a location close to the central ionizing source. There are two obvious sites for the X-ray absorber: the clouds in the Broad Line Region (BLR); and the clouds responsible for the high ionization UV absorption lines.

\subsection{BELR Clouds as X-RAY Absorber?}

Standard photoionization models for the BLR imply U (0.01) more than two orders of magnitude smaller than the best fit value for X-ray Absorber. However, reverberation studies (Peterson, 1988) have shown than the BLR is small and stratified so that parts of the BLR have higher $U$ than previously thought. In addition, $3 \mathrm{C} 351$ is $\mathrm{X}$-ray quiet, requiring higher $\mathrm{U}$ to produce the observed emission lines.

T. J.-L. Courvoisier and A. Blecha: Multi-Wavelength Continuum Emission of AGN, 271-274.

(C) 1994 IAU. Printed in the Netherlands. 

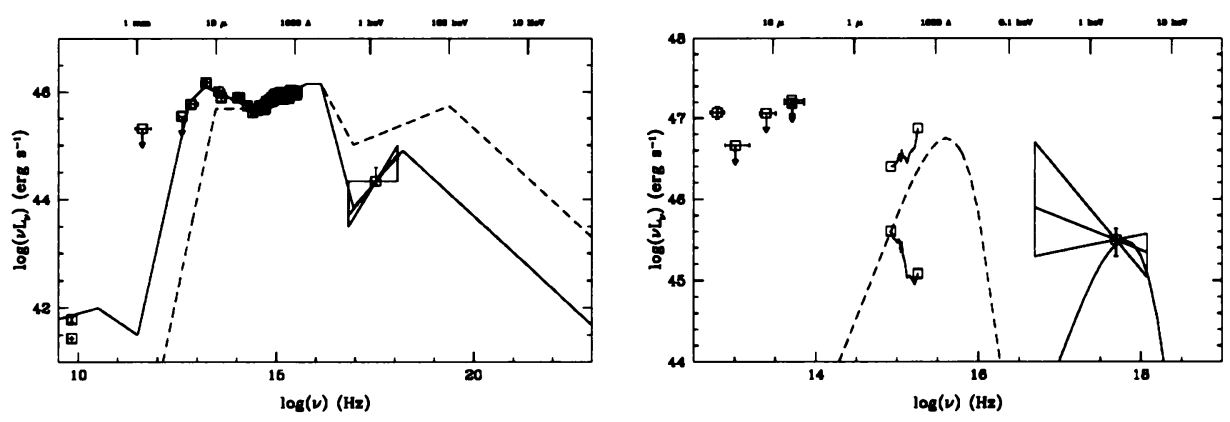

Fig. 1. SEDs for (a)3C351: - = the best fit SED, $\ldots=$ = 'average' quasar continuum for radio-loud quasars. (b)3C212: see the text. - - - =typical quasar UV bump.

The observed SED has a large effect on the strengths of high ionization emission lines (using CLOUDY, Ferland, 1991): OVI 1034 is weakened by almost an order of magnitude (Figure 2a). The model is compared with the observed (HST spectrum) line ratios. Even though the inferred $U$ for OVI line is as high as 0.3 , it's still much lower than that required for the X-ray absorber. So the BELR is not a plausible site for the X-ray ionized absorber. The X-ray quiet SED also affects traditional density diagnostics; CIII] $\lambda 1909$ ceases to be a density indicator for this SED (Figure 2b) (Mathur et al, 1994) due to increased free-free heating which produces heating without ionization.

\subsection{X-RAY Absorber as UV Absorber?}

The ultraviolet HST spectrum of 3C351 (Bahcall et al 1993) shows an unusually strong associated metal line absorption system. In particular, strong OVI absorption doublets are observed (c.f. OVII absorption edge in soft X-rays).

Table 1.

\begin{tabular}{|c|c|c|}
\hline $\begin{array}{c}\text { Inferred Parameter } \\
\text { from HST Spectrum }\end{array}$ & \multicolumn{2}{|c|}{ Model Parameter } \\
\hline$f_{O V I}=-1.52$ & -1.532 & -1.526 \\
$f_{N V}=-2.86$ & -2.43 & -2.42 \\
$f_{C I V}=-2.81$ & -2.79 & -2.8 \\
\hline
\end{tabular}

X-ray observations give strong constraints on the total $N_{H}=1.4 \times 10^{22} \mathrm{~cm}^{-2}$ which allows us to solve for a consistent model of UV lines and X-ray absorber. The UV absorption lines give the following constraints: $N_{H}>2 \times 10^{18} / f_{(O V I)} \mathrm{cm}^{-2}$ (where $f_{(O V I)}$ is the ionization fraction of oxygen in OVI state), ' $b$ ' parameter $<600-1200 \mathrm{~km} \mathrm{~s}^{-1}$ (= FWHM of the lines). A small value $\left(\sim 4 \mathrm{~km} \mathrm{~s}^{-1}\right)$ of 'b' implies unacceptably large column density. We find that ' $b$ ' $110 \mathrm{~km} \mathrm{~s}^{-1}$ satisfies 

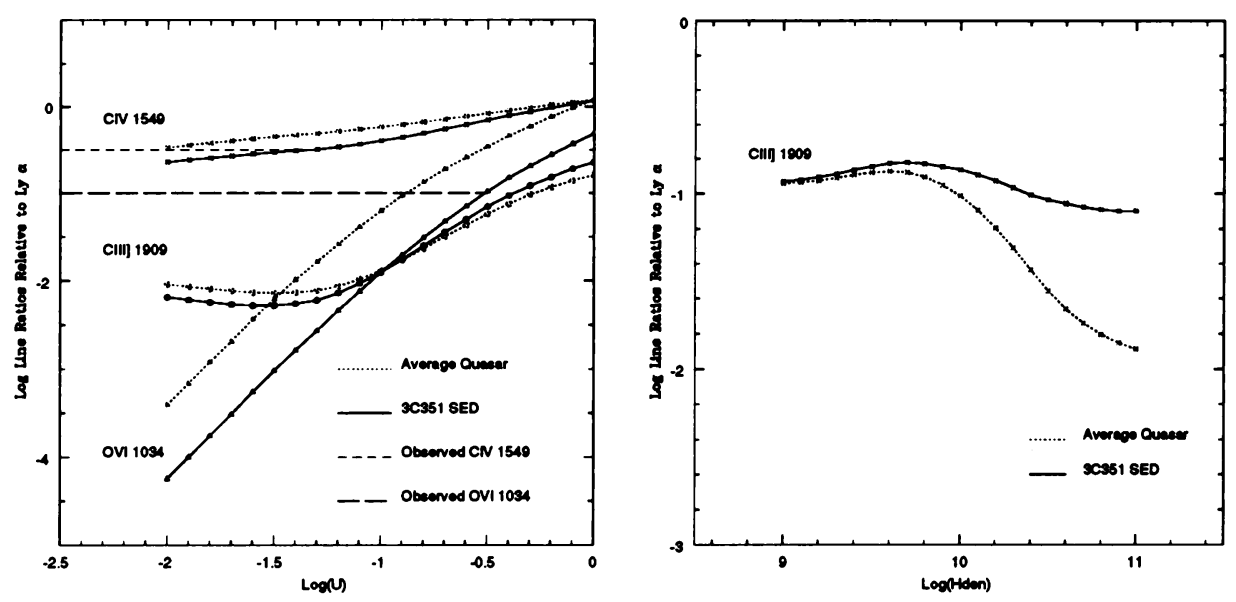

Fig. 2. Emission line strengths relative to $L y \alpha$ as a function of (a) ionization parameter (b) density. Errors on observed line strengths are $\sim 10 \%$

the constraints on the ionization fractions of all the high ionization absorption lines; OVI, CIV and NV and allows a consistent model (Table 1 ) for $U=6.7$. We conclude that the identity of X-ray absorber as the UV absorber is likely, although we cannot rule out two separate absorbers (Mathur et al 1994). The implied physical properties of the X-ray and UV absorber are: high $N_{H}\left(1.4 \times 10^{22} \mathrm{~cm}^{-2}\right)$, high $\mathrm{U}$ (6.7), an outflow velocity of $\sim 2000 \mathrm{~km} \mathrm{~s}^{-1}$ (from UV blueshift), low density $\left(10^{3}-10^{5} \mathrm{~cm}^{-3}\right)$, large size $(\sim 10-0.1 p c)$ and distance from the central source $(\sim$ $100-10 p c)$, low covering factor $(<0.04)$, and mass loss rate $2<\dot{\mathrm{M}}<20 M_{\odot} y r^{-1}$. This implies kinetic luminosity of $\sim 10^{42}-10^{43} \mathrm{ergs}^{-1}$, which is only $\sim 10^{-3}-10^{-4}$ of the radiative luminosity of the quasar. These properties describe a component of nuclear material not previously recognized.

\section{3C212: A Red Quasar}

The extreme case of a UV weak ionizing continuum may be seen in the case of the 'Red Quasars' (Smith \& Spinrad 1980). Figure 1b. shows the SED for 3C212 which could either have a red optical-UV continuum and a X-ray black body or a dereddened optical-UV and X-ray power law (Elvis et al 1994). Clearly, the two models have extreme differences in the implied shape of their UV to soft X-ray continuum, with a factor of $\sim 10^{3}$ difference in the number of ionizing photons. They might be expected to predict quite different emission line ratios.

Surprisingly, in spite of these huge differences between the possible ionizing continua, the photoionization predictions provide no convincing discriminants between the models given the few observed lines. The similarity of the predictions is partly because the increased number of ionizing photons in the de-reddened power law is almost exactly matched by the increased number of emitted line photons, since 
both lines and continuum are dereddened by the same amount. Another limitation is that there are few observed lines to provide constraints. Only Mg II $\lambda 2798 \AA$ and C III] $\lambda 1909$ are clearly detected (Smith \& Spinrad 1980). All the Hydrogen lines lie outside the observed range $(1500 \AA-3560 \AA$, rest). Better diagnostics could be searched for with improved optical and infrared spectra . For example, the greatest difference between the two continua is in the EUV, so that the HeII lines, which require $\lambda<304 \AA$ photons for their production, should provide a clean discriminant between the two models.

\section{Conclusions}

An X-ray quiet SED, as in 3C351, strongly affects the governing parameters in the BELR physics. We identify the X-ray absorber in 3C351 as the UV absorber. This implies a new component of the nuclear material. The 3C212 continuum could either be red optical-UV and a X-ray black body; or a dereddened optical-UV and $\mathrm{X}$-ray power law. Observations of HeII lines could discriminate between the two models. The strategy of seeking out extreme quasars does produce new insights. The study of "Quasars across electromagnetic spectrum" is indeed the key towards understanding the underlying physical processes.

\section{Acknowledgements}

Fabrizio Fiore, Martin Elvis and Belinda Wilkes have contributed to the present investigation. This work was supported by NASA grant NAGW-2201.

\section{References}

Bahcall, J. N. et al 1993, Ap.J.Supp., 87, 1.

Elvis, M., Fiore, F., Mathur, S., Wilkes, B., 1994, Ap.J., submitted.

Ferland, G. F. 1991 OSU Astronomy Department Internal Report.

Fiore, F., Elvis, M., Mathur, S., Wilkes, B. J., McDowell, J. C. 1993, Ap.J., 415, 129.

Mathur, S. et al 1994, in preparation.

Peterson, B. M. 1988, P.A.S.P., 100, 18.

Smith, H. E. \& Spinrad, H. 1980, Ap.J., 236, 419. 